\title{
Interactions between simultaneous aerobic exercise and mental rotation
}

\author{
Leonardo Jost ${ }^{1}$ (D) $\cdot$ Andreas Weishäupl ${ }^{2} \cdot$ Petra Jansen $^{1}$
}

Accepted: 21 April 2021 / Published online: 8 May 2021

(C) The Author(s) 2021

\begin{abstract}
While the effects of aerobic exercise during a cognitive task on the performance of said cognitive task have been extensively studied, it has not been investigated whether cognitive performance during aerobic exercise influences the physical performance. For this, it is the main goal of the study to investigate the physical and cognitive performance during a simultaneous conduction of aerobic exercise and mental rotation. Forty-one German sport students cycled at $60 \%$ intensity while simultaneously performing a mental rotation task. In a within-subject design, both physical and cognitive performances were compared with isolated cycling and mental rotation as control conditions using both objective (heart rate and pedal cadence in the cycling task, reaction time and accuracy in the mental rotation task) and subjective (RPE) cognitive and physical measures. The results analyzed with hierarchical linear modeling revealed no effect of either simultaneous cognitive tasks on objective (heart rate) or subjective (RPE) physical effort, nor of simultaneous exercise on reaction time or accuracy in cognitive performance. However, we have found lower cadence during cognitive tasks, which was also stable in time compared to an increase in cadence during exercise control. Furthermore, our results demonstrated increased cognitive effort during exercise. Our findings suggest that increased effort, both physiological and cognitive, is required during combined physical and cognitive work in support of neurological resource conflicts caused by the differing demands of exercise and executive function.
\end{abstract}

Keywords Mental rotation $\cdot$ Executive function $\cdot$ Aerobic exercise $\cdot$ Subjective effort $\cdot$ Spatial cognition

\section{Introduction}

A large body of research has shown that not only chronic but also acute aerobic exercise influences cognitive functions (Chang et al., 2012; Lambourne \& Tomporowski, 2010; Oberste et al., 2019). On the other hand, the effects of cognitive activity on physical performance have also been analyzed, but the effect on simultaneously performed aerobic exercise has, to our knowledge, not been a topic of research. This might be relevant for endurance athletes who want to use their training time more effectively. If cognitive learning is possible during endurance training, leisure time and/or academic success can be increased. Furthermore, this analysis of acute effects is also important for the implementation of devices such

Leonardo Jost

Leonardo.jost@ur.de

1 Faculty of Human Sciences, University of Regensburg, Universitätsstraße 31, 93053 Regensburg, Germany

2 Sports Centre, University of Passau, Passau, Germany as treadmill desks to promote physical activity. Having both beneficial effects in mind, namely the possible time saving as well as the promotion of physical activity, it is the main goal of this study to investigate the effect of aerobic exercise while solving a difficult cognitive task, a mental rotation task, and vice versa the effect of solving a mental rotation task on physical performance. Mental rotation was chosen as a cognitive task because it is related to academic achievement, for example mathematical abilities (Xie et al., 2020) or STEM education in general (Moè et al., 2018).

\section{The Effects of Performing a Cognitive Task while Exercising on Exercise Performance}

An analysis of the effects of performing cognitive tasks while exercising on aerobic performance has not yet been conducted. What has been investigated is a) the effect of exercise on cognitive performance, with cognitive performance measured during and after the exercise bout and b) the interaction of simultaneous cognitive tasks and cognitively demanding motor tasks in dual-task paradigms. 
Regarding the effect of acute aerobic activity on cognitive tasks, Lambourne and Tomporowski (2010) revealed in their meta-analysis that overall exercise has a small detrimental effect on cognitive performance during the exercise. These effects were dependent on the type of exercise: during running, the negative effects were larger whereas during cycling, the effects were even positive, although possibly not significant. In contrast, in another meta-analysis, Chang et al. (2012) found overall small enhancing effects of exercise on simultaneous cognitive performance. In both meta-analyses, the time of cognitive testing, the type of cognitive task (e.g. executive functions), and the exercise intensity were identified as moderators. However, the results were not unanimous whether effects during the first $10 \mathrm{~min}$ of exercise were negative or negligible; effects after 11-20 min of exercise were negative but positive later on. Chang et al. found the largest effects for tests of executive functions, which included working memory, inhibition, and cognitive flexibility, whereas Lambourne and Tomporowski identified no effect for tests of executive functions. Regarding intensity, both found the largest positive effects for moderate exercise. The analysis of the fitness level of participants by Chang et al. also revealed differences. Positive effects were only evident for highly fit participants compared to no effects with moderately fit participants and negative effects for unfit participants. Chang et al. explained the contrasting results compared to Lambourne and Tomporowski with different inclusion criteria. Whereas Lambourne and Tomporowski integrated only studies that tested young and healthy participants and used a repeated measures design, Chang et al. included studies with participants of all ages and the use of any design. In a recent systematic review and meta-analysis, Oberste et al. (2019) analyzed the effect of an acute bout of aerobic exercise on subsequent tasks of interference control. Their results demonstrated that aerobic exercise improves subsequent cognitive performance. This effect was larger for participants at younger (preadolescent and adolescent) or older age, for participants who were familiar with the procedure, and after exercises of high intensities.

Dual-task studies of simultaneous exercise and cognitive tasks demonstrated that the stability and accuracy of cognitively demanding motor tasks is affected, e.g. balance and gait behavior for walking in older adults. However, the physiological performance of automated motor tasks has not been investigated. Dual-task costs generally describe impairments under dual-task conditions, although facilitations are sometimes also observed (e.g. Schaefer et al., 2015; Schaefer \& Schumacher, 2011).

Next to the aforementioned studies, other work indicates that mental fatigue induced by a cognitive task prior to exercise increases the subjective perception of effort, but objective physiological parameters remain unaffected (Martin et al., 2018; Van Cutsem et al., 2017). Moreover, physiological parameters including heart rate are affected by cognitive tasks in resting conditions (Kahneman et al., 1969; Kennedy \& Scholey, 2000) and these effects might persist during exercise. This indicates that mental processes may influence aerobic performance exceeding motivation.

\section{Models to Explain the Effects of Physical Exercise on Cognitive Tasks}

The effects of physical exercise on cognitive tasks can be explained by different models: a) the reticular activating hypofrontality model (RAH, Dietrich \& Audiffren, 2011) and $b$ ) the strength model (Audiffren \& André, 2015; Baumeister et al., 1998, 2007). The RAH states that automated motor tasks used in aerobic exercise involuntarily lead to hypofrontality and reticular activation due to the evolutionary optimization of movement control and limited neural resources. This in turn leads to improvements in the implicit system, which is experience-based and inaccessible to conscious awareness and to a decline in the explicit system, which is rule-based and tied to conscious awareness. For simultaneous cognitive tasks, this predicts improvements for more implicitly driven automated tasks and impairments for tasks requiring explicit attention such as visual working memory tasks. In the strength model, both exercise and executive functions require the limited resource of self-control. If self-control resources are depleted by exercise, performance in effortful cognitive tasks should deteriorate. While the strength model is undirected as to which activity drains self-control and thus would also predict a decline in exercise performance if selfcontrol is depleted after the use of executive functions, the RAH model in theory only analyzes the effect of exercise on cognitive performance. However, as executive functions are associated with brain activity which opposes the exercise facilitating brain activity proposed by the RAH model, a decline in physical performance seems feasible. This partially aligns with the results of the aforementioned meta-analyses and dualtask studies; as cognitive tasks become more complex, they might require more effort from the explicit system in line with larger observed dual-task costs. Similarly, as motor tasks become more cognitively demanding due to age or less demanding due to a higher fitness level, they might require more or less effort from the explicit system. Both models would predict increased dual-task costs in the elderly and lower cognitive performance in less fit individuals, as is typically observed. Nevertheless, there exists contradicting evidence for the models, both in the predictions of cognitive outcome measures as in part in the aforementioned meta-analyses (Chang et al., 2012; Lambourne \& Tomporowski, 2010), as well as in measurements of brain activity (e.g. Dodwell et al., 2019). For a visual working memory task during moderate aerobic activity on a treadmill and a bicycle, Dodwell et al. found better performance compared to seated and standing control conditions. Using EEG, they also failed to find evidence for neural 
resource conflicts and even facilitation during processing stages, which should require the explicit system. However, it is possible that the cognitive tasks were too easy and performance was not hindered by the neural resource reallocations predicted by the RAH. If performance is not limited by neural resources, overall performance might be more influenced by how much effort participants are willing to invest, either consciously or subconsciously. Dodwell et al. further stated that performance facilitation might also occur due to differences in overall arousal, which is not a subject of the RAH. The larger facilitation in the standing and running conditions might also be due to an overlap of neural mechanisms governing balance and spatial cognition. This suggests that neural resource allocation does indeed influence performance but the RAH does not account for these overlaps.

\section{The Effects of Exercise on Mental Rotation}

Numerous studies have investigated the relation between physical activity and simultaneously solving an executive function task (see Chang et al., 2012). We decided to examine the relation of physical activity with mental rotation, namely the ability to imagine three-dimensional objects (Shepard \& Metzler, 1971), because this spatial task is more complex and related to physical exercise. In previous studies, it has been assumed that mental rotation is a covert motor rotation (Wohlschläger \& Wohlschläger, 1998) and that during mental rotation motor areas are activated (Jordan et al., 2002). Because of this assumption, it is not unusual for sessions of physical exercise to demonstrate the capacity to improve mental rotation performance for both children (Pietsch et al., 2017) and adults (Jansen et al., 2009). Furthermore, enhancing effects have also been shown after acute exercise (Jansen \& Pietsch, 2010) in line with the enhancing effects found by Chang et al. (2012) and Lambourne and Tomporowski (2010) for different cognitive tasks following exercise, but we have found no studies analyzing mental rotation performance during exercise. Moreover, facilitation has also been recognized for chronic exercise (Jansen et al., 2012; Voyer \& Jansen, 2017). However, it is not clear how and if these transfer to acute exercise.

Mental rotation can be investigated using chronometric tests with different types of stimuli. In chronometric objectbased mental rotation tasks, participants see two objects on a screen, which are rotated from each other and might be mirrored or non-mirrored. Participants must decide as quickly and accurately as possible whether the two objects are the same (non-mirrored) or different (mirrored) (Shepard \& Metzler, 1971). In mental rotation tests, the effect of sex is widely discussed. Whereas in the seminal meta-analysis of Voyer et al. (1995) a male advantage is detected in psychometric mental rotation tasks, Jansen-Osmann and Heil (2007) showed that this male advantage only holds true for one type of stimuli out of five in a chronometric mental rotation test.

\section{Study Goal}

It is the main goal of this study to explore the possible influences of mental rotation on physical exercise, as well as the effect of physical exercise on mental rotation while also analyzing subjective effort by measuring the rating of perceived exertion (RPE). Mental rotation was chosen as a complex cognitive task in which different stages are used for processing: the perceptual stages (perceptual processing, identification and discrimination of stimuli, identification of orientation); stages of the rotation process itself (mental rotation, judgment of parity); and decision processing stages (response selection, execution) (Heil \& Rolke, 2002). To complete these stages, components of working memory are necessary (Hyun \& Luck, 2007), and their relation to physical performance has been shown. However, no study has investigated the simultaneous conduction of a physical activity as well as a mental rotation task as a rather complex working memory task.

To this end, we will compare the simultaneous performance of cycling and mental rotation to both isolated cycling at the same power output and isolated mental rotation conditions. According to both the RAH and the strength model, negative effects in both directions are expected, i.e. an increased heart rate and slower and less accurate responses for the mental rotation task as well as larger RPEs. Additionally, with increasing exhaustion during the experimental task, we expected these effects to increase over time.

\section{Methods}

\section{Participants}

For the power analysis, Brysbaert and Stevens (2018) suggest using the typical effect sizes in psychology of $d=0.4$ or $d=$ 0.3. For effect sizes of $d=0.4, G^{*}$ power (Faul et al., 2007) reveals a power of 0.81 for within-subject comparisons of 41 participants, which should further increase with the use of linear mixed models (Barr et al., 2013; Hilbert et al., 2019). With the planned duration of the mental rotation tasks of $30 \mathrm{~min}$ and an estimated average reaction time of $3 \mathrm{~s},{ }^{1}$ this should also exceed the recommendation of Brysbaert and Stevens (2018) for analyses of reaction times of at least 1600 observations per condition to detect commonly observed very small effect sizes of around $d=0.1$. For the effect of exercise on cognitive performance, Chang et al. (2012) report effect sizes of $d=0.10$ during exercise, moderated by intensity

\footnotetext{
${ }^{1}$ As only correct answers on rotated trials are analyzed, we expected such a trial once in about $10 \mathrm{~s}$ or about 180 such trials per participant over $30 \mathrm{~min}$.
} 
(moderate intensity with $d=0.19$ ), task type (executive function as the most comparable category with $d=0.26$ ), and fitness level (moderate fitness level with $d=0.01$ ). As we expect much lower variance in physiological measures within participants, the estimated effect sizes of $d=0.4$ and $d=0.1$ seem appropriate.

Accordingly, a total of 41 German sport students (22 women, 19 men) participated in this study. Participants were recruited through a seminar as part of which the maximal performance tests were performed and received additional study credit. Participants chose the day of the week and the time of the day on a first come, first served basis. Starting times were available from 9:00 to $12: 00$ and $13: 45$ to $17: 30$ in 45 -min slots from Monday to Friday. Two additional students wanted to participate but had to withdraw prior to the first session, one due to illness and one due to timing conflicts. All participants reported no limitations regarding physical exercise or digitally presented cognitive tasks. Participants were instructed to continue with their usual eating, sleeping, and training habits but not to perform additional training on the same day prior to testing. Self-reported demographic data is summarized in Table 1.

\section{Material}

\section{Cognitive Measures (Chronometric Mental Rotation Test)}

Stimulus presentation and response handling were controlled with Presentation ${ }^{\circledR}$ software (Version 20.1 Build 12.04.17, Neurobehavioral Systems, Inc., Berkeley, CA) on a Dell Latitude E7240 Laptop and presented on an external 22 in. Dell 2208WFP monitor, $1366 \times 768,59 \mathrm{~Hz}$, positioned approximately $50 \mathrm{~cm}$ in front of the handlebars independent of participants' size in the experimental sessions and $50 \mathrm{~cm}$ in front of a chair in the practice session.

Table 1 Participants' Data

\begin{tabular}{lll}
\hline Variable & Men & Women \\
\hline $\mathrm{n}$ & 19 & 22 \\
Age (years) & $22.7(2.35)$ & $20.1(1.58)$ \\
Height $(\mathrm{cm})^{*}$ & $181.1(6.35)$ & $166.2(5.16)$ \\
Weight $(\mathrm{kg})^{*}$ & $79.3(13.19)$ & $60.0(7.10)$ \\
Physical activity (h/week)* & $9.8(4.12)$ & $7.0(4.34)$ \\
Handedness & $5.4(6.79)$ & $8.4(2.65)$ \\
Maximal power $(\mathrm{W})^{*}$ & $273.4(39.79)$ & $178.9(23.35)$ \\
Relative maximal power (W/kg)* & $3.5(0.56)$ & $3.0(0.45)$ \\
Maximal heart rate (bpm) & $188.1(10.16)$ & $189.8(10.13)$ \\
Time until exhaustion $(\mathrm{min})^{*}$ & $16.8(3.37)$ & $11.9(2.33)$ \\
\hline
\end{tabular}

Note. Mean (SD) of participants anthropometric and physiological data, separated by sex. Significant differences $(p<.05)$ by sex are marked by *
Stimuli were obtained from the stimulus library of Peters and Battista (2008). All 16 models with a rotation around $\mathrm{x}$ - and $\mathrm{z}$ axis in $45^{\circ}$ steps and mirrored/non-mirrored orientations ( $\mathrm{a}$ and $\mathrm{b}$ orientation in the stimulus library) were used with a checkered pattern on a black background, resulting in a total of 480 different stimuli. On the left side of the screen the model was presented in orientation a, rotated by $30^{\circ}$ in $\mathrm{x}$ direction and $15^{\circ}$ in $\mathrm{z}$ direction, such that the base model for $\mathrm{x}$ or $\mathrm{z}$ rotations was identical. On the right side of the screen, a rotated and mirrored/non-mirrored stimulus was presented. Stimulus pictures were sized 400px times $400 \mathrm{px}$ and presented vertically centered and horizontally positioned 300px to the left or right of the center of the screen (see Fig. 1) until a response was given. Between stimulus pairs in the practice session, participants received feedback for $1000 \mathrm{~ms}(\boldsymbol{\checkmark}$ right, $\boldsymbol{X}$-wrong) displayed in the center of the screen at font size 40 , in experimental sessions a fixation cross (" + ") was shown at the center of the screen for $500 \mathrm{~ms}$.

Stimuli were presented in a predefined random order until either $10 \mathrm{~min}$ (practice session) or $30 \mathrm{~min}$ (experimental sessions) had passed. Three orders of stimulus pairs were randomly generated using shuffling in Presentation ${ }^{\circledR}$ software, such that all stimuli were shown once in random order before they were shown again. Between two occurrences of the same stimulus pair, at least 30 other stimulus pairs were shown. In the practice session, the same order was used for all participants. However, for the two experimental sessions, the remaining two orders were assigned randomly, counter-balanced between participants and the order of experimental sessions using random permutations in R (R Core Team, 2018).

In addition to the stimulus pairs, total trial duration was presented in $15 \mathrm{~s}$ steps at the top of the screen (horizontally centered, 300px above the center of the screen, font size 48), albeit only updated when the screen changed between stimuli, not while one stimulus pair was shown.

Participants were digitally instructed to press the left mouse button if the stimuli could be rotated into congruence (non-mirrored), and the right mouse button if the two stimuli were mirrored and to answer as quickly and as precisely as possible. Mouse handling was not specified. Thirty-five participants used their right hand and kept it constantly on the mouse in all sessions, whereas six participants used different mouse handling but used similar handling in both experimental sessions involving mental rotation.

Reaction time, accuracy, and stimulus type (model, rotation angle, rotation axis, orientation), as well as time since the start of the trial, were recorded.

\section{Physiological Measures}

Cycling was performed on a Cyclus2 ergometer (RBM elektronik-automation $\mathrm{GmbH}$, Leipzig, Germany) allowing control of power independent of pedal cadence with a racing bicycle frame and racing saddle (Cube Peloton, Size S). Saddle height was adjusted to participants individually, such 


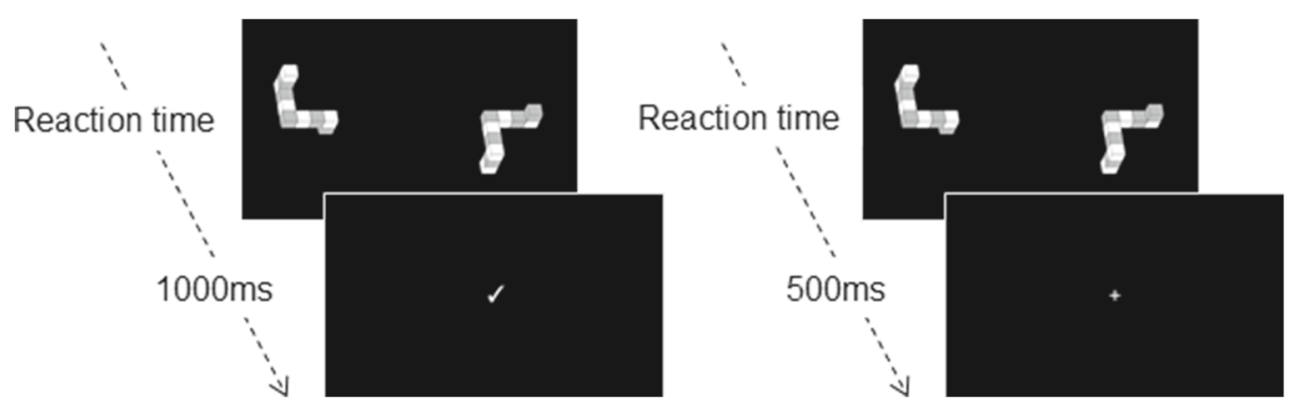

Note. Structure of a mental rotation trial. First, participants performed the mental rotation task with unlimited time until they gave a response. In the practice session, they then received feedback ( $\checkmark$-right, $\boldsymbol{X}$-wrong) for 1000ms (left figure). In the experimental sessions, they saw a fixation cross $(+)$ for $500 m s$ (right figure). The stimulus pair is an example of a rotation around the $x$-axis by $180^{\circ}$.

Fig. 1 Mental Rotation

that they were able to cycle comfortably, although handlebar height remained constant for all participants. Participants were instructed to cycle at a cadence of their choice, but at least $50 \mathrm{rpm}$. If it dropped below $50 \mathrm{rpm}$, participants were asked to increase their cadence (only applied to one participant in the only exercise condition). Heart rate was measured using a heart rate belt (Polar $\mathrm{H} 7$ or Cosmed) and was transferred to a watch (Polar M400) or Cyclus2.

Heart rate was documented every $5 \mathrm{~min}$ in all experimental sessions and pedal cadence in all experimental sessions involving exercise.

\section{Subjective Effort}

Both subjective physical effort (physical rating of perceived exertion, pRPE) and subjective cognitive effort (cognitive rating of perceived exertion, cRPE) were measured using the "Anstrengungsskala Sport" (ASS), an RPE scale developed in German by Büsch et al. (2015) ranging from 0 to 10 . The scale was shown to participants and they were asked to name a unique number or word description describing their physical or cognitive effort. In the experimental sessions, RPE measurement followed directly after finishing the exercise/tasks. In the case of simultaneous physical and cognitive tasks, pRPE was measured first.

\section{Procedure}

This study employed a cross over design and participants were required to visit the laboratory on four occasions (one preexamination and practice session and three experimental sessions) a week apart on both the same day of week and same time of day. Twenty-nine participants fulfilled these timing requirements, ten participants differed from this rhythm, but had at least $48 \mathrm{~h}$ of rest between sessions; two participants only managed to participate on three occasions. Temperature, humidity, light, and sound conditions were not controlled for.
In the pre-examination and practice session, participants were informed about the study design and goals and their individual order of experimental sessions and were also instructed about the study's usage of the ASS. An incrementing stage test was performed to exhaustion with an increase of $30 \mathrm{~W}$ every $3 \mathrm{~min}$. Starting power for women was set to $90 \mathrm{~W}$, for men weighing less than $80 \mathrm{~kg}$ to $120 \mathrm{~W}$, and for men weighing at least $80 \mathrm{~kg}$ to $150 \mathrm{~W}$. For one selfreported competitive endurance-athlete, the starting power was increased by a further $30 \mathrm{~W}$. This is in line with some commonly applied test protocols with consideration for varying absolute power output by sex, weight, and endurance fitness level, but there exists no consensus on test protocols (Bentley et al., 2007; Faria et al., 2005b). The end of the test was determined by subjective exhaustion (an RPE of 10) despite strong verbal encouragement. RPE was measured in the last 10 s of every stage for habituation purposes. Heart rate and pedal cadence were continuously measured and could be seen by participants. Maximal heart rate and maximal power were documented, where maximal power was calculated as the power of the last completed stage plus $5 \mathrm{~W}$ for every additional $30 \mathrm{~s}$ completed of the next stage to adjust for the proportion of the last stage (Hopkins et al., 2001). Maximal heart rates exceeded $95 \%$ of participants' age predicted peak heart rate (200 - age; Such \& Meyer, 2010), indicating that all participants achieved exhaustion objectively.

After finishing the performance test, participants were allowed to rest until they felt ready to continue with the practice session of 10 min of mental rotation. To reduce familiarization with the experimental conditions, participants were seated in a chair for the practice session. At the end of the first session, cRPE, age, height, weight, physical activity (the sum of aerobic activity, weight training, and sports games), and handedness according to the Edinburgh handedness inventory (Laterality quotient ranges from -10 to +10 ; Oldfield, 1971) were measured using a digital questionnaire. 
All experimental conditions lasted for $30 \mathrm{~min}$. In the exercise only (E) and the mental rotation while exercising (ME) conditions, participants cycled at $60 \%$ of their individual maximal power, rounded to $5 \mathrm{~W}$. Individual maximal power has shown to be a good predictor of aerobic cycling performance and $60 \%$ corresponds to a moderate intensity range, such that all participants could cycle at this intensity for $30 \mathrm{~min}$ without premature exhaustion. An intensity prescription using blood lactate thresholds was avoided as invasive methods have to be used and small variations in intensities should have a limited effect on cognitive performance.

The duration was set to $30 \mathrm{~min}$, as this is generally used to evaluate lactate steady states and should thus be sufficient to reach valid measurements of heart rate and RPE; it also matches or exceeds duration recommendations for aerobic activity (Bentley et al., 2007; Faria et al., 2005b; Hohmann et al., 2002). In the mental rotation only (M) condition, participants sat on the bicycle frame without cycling. Participants had no knowledge of their power output, pedal cadence, or heart rate, but were informed that power was kept constant over the duration thereof. In the E condition, participants were informed every $5 \mathrm{~min}$ about the time, otherwise there was no interaction between conductor and participant and conductors stayed out of participants' visual field.

Additionally, before the last experimental session, baseline heart rate was measured after adjusting the ergometer and participants stayed seated but rested on the ergometer for $30 \mathrm{~s}$.

\section{Study Design}

Three experimental conditions were used: exercise only (E), mental rotation only $(\mathrm{M})$, and mental rotation while exercising (ME). The $\mathrm{M}$ condition was always performed in the second experimental session, whereas $\mathrm{E}$ and $\mathrm{ME}$ were performed in random order in the first and third experimental sessions, counter-balanced between participants and the random orders used for mental rotation, such that all participants had two weeks of rest between physical tests and one week of rest between cognitive tests.

For cognitive performance, dependent variables were reaction time, accuracy, and cRPE. For physical performance, dependent variables were pedal cadence, heart rate, and $\mathrm{pRPE}$. Independent variables were condition (M and $\mathrm{ME}$ for cognitive performance and $\mathrm{E}$ and $\mathrm{ME}$ for physical performance), time (since start of the session), the interaction between condition and time, sex, and for cognitive performance also the angle of rotation.

\section{Statistical Analysis}

Statistical analysis was performed with linear mixed models using the lme4 package (Bates et al., 2015b) in R (R Core Team, 2018). Model parameters were estimated by maximum likelihood estimation. $P$-values were obtained by using likelihood ratio tests to test for improvement of model fit by the fixed effect of interest and compared to a significance level of 0.05 . Confidence intervals were calculated using parametric bootstrapping with 1000 simulations. A visual inspection of residual plots did not reveal any deviations from homoscedasticity or normality in any model.

Where possible, we report both the unstandardized effect sizes and confidence intervals calculated by using parametric bootstrapping with 1000 simulations in line with the recommendations of Baguley (2009) and Pek and Flora (2018). While standardized effect sizes are routinely used for power analysis and meta analyses, unfortunately there is not a consensus regarding how to compute standardized effect sizes in linear mixed models (Feingold, 2009; Hedges, 2007; Rights \& Sterba, 2019). Nevertheless, linear mixed models offer several advantages over traditional ANOVAs. For example, linear mixed models allow the simultaneous analysis of byparticipant and by-item variances, thus eliminating the need to average over participants or items, while also facilitating the analysis of unbalanced data and achieving higher statistical power (Barr et al., 2013; Hilbert et al., 2019).

Model building was based on the research of Barr et al. (2013) and Bates et al. (2015a), starting with a model with random intercepts and slopes for every appropriate fixed effect and subsequently reducing the model complexity by dropping non-significant variance components. Nonsignificant fixed effects were further removed from the model, such that non-significant effects were tested for an improvement of model fit by inclusion in the resulting model, while significant effects were tested for worsening of model fit by exclusion of the effect. The resulting models for each parameter are described in the results section.

As there is ongoing discussion about model selection based on theory or data or preferring complex instead of simpler models, we expect future research to cast doubt on the optimality of the currently suggested models. Although models without random slopes seem too anti-conservative (Barr et al., 2013), we report the results of these simplest models for easier comparison.

As it is not clear whether RPE values should be treated as interval or ordinal (e.g. Bishop \& Herron, 2015), all statistical analyses of RPE values have been conducted twice, with one treating the values as ordinal and one as interval. No differences in significance were found in any analysis and we report only the results obtained by treating the values as interval scaled.

\section{Results}

\section{Physical Performance}

\section{Pre-Examination Data}

The results of the performance test and questionnaire are presented in Table 1. Most notably, we found differences by sex 
in physical activity and time until exhaustion in the performance test with longer test duration for men.

\section{Heart Rate during Exercise}

For the analysis of heart rates, the model building resulted in a model with a random intercept and random slopes for condition and time by participant. Condition, sex, time, and the interaction between condition and time were analyzed as fixed effects; in doing so, significant effects were found for sex, time, and condition*time but not for condition (see Table 2 for inferential statistics and Fig. 2 for the descriptive comparison of conditions over time). Heart rate increased significantly with time and women showed significantly higher heart rates; however, differences between conditions were not significant. The point estimate of less than $1 \mathrm{bpm}$ indicates no meaningful overall difference between conditions. The analysis of the interaction showed a significantly lower intercept and larger increase in heart rate in the $\mathrm{E}$ condition, while differences at our measured time points were only significant at the 30-min mark.

\section{Heart Rate without Exercise}

In the analysis of time in the $\mathrm{M}$ condition, random intercepts and slopes for time by participants were used. To compare with baseline and analysis of sex, random intercepts and slopes for condition by participants were used. Condition, time, and sex were analyzed as fixed effects and no significant effects were found (see Table 3). However, in a model without random slopes by time the increase of heart rate by time was significant $(p=0.01)$.

\section{Pedal Cadence}

For the analysis of pedal cadence, the model building resulted in a model with random intercepts and random slopes for

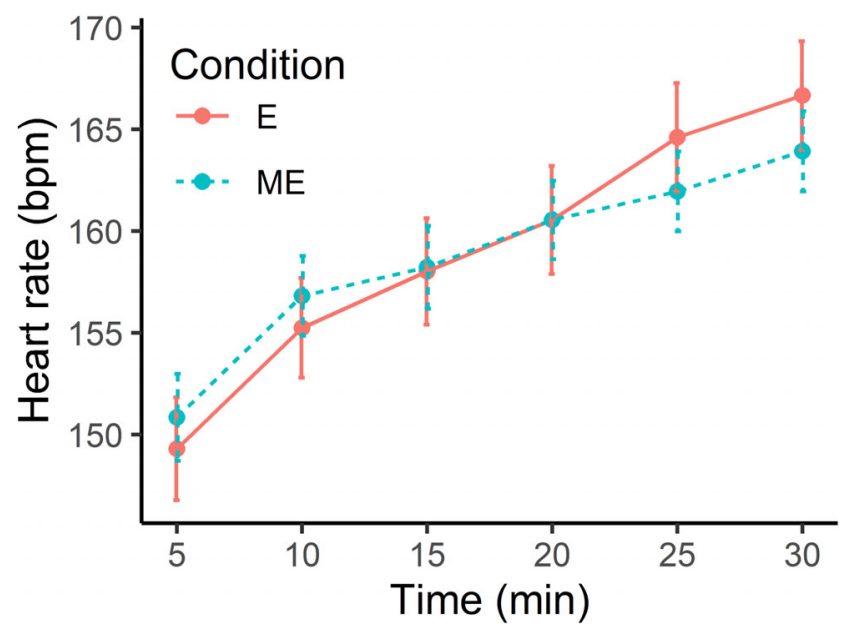

Note. Line plots showing mean heart rate as a function of time for both exercise conditions. Error bars show standard error. E - only exercise condition, $M E$ - combined mental rotation and exercise condition.

Fig. 2 Heart Rate During Exercise

condition and time by participants. Condition, sex, time, and condition*time were analyzed as fixed effects and significant effects were found for condition, time, and condition*time (see Table 4 for inferential statistics and Fig. 3 for the descriptive comparison of conditions over time). Pedal cadence was significantly lower in the ME condition by an estimated $8.32 \mathrm{rpm}$ and increased significantly over time. The analysis of the interaction between time and condition revealed a significant increase with time only in the E condition, but not in its ME counterpart. Pedal cadence did not differ significantly at $5 \mathrm{~min}$, but all later times showed significantly higher cadences in the E condition.

\section{Physical Subjective Effort}

For the analysis of pRPE, the model building resulted in a model with only a random intercept by participant. Condition and sex were analyzed as fixed effects and neither
Table 2 Statistical Analysis of Heart Rate During Exercise

\begin{tabular}{llllll}
\hline Variable & Estimate & SE & Test statistic & $\mathrm{p}$ & $95 \% \mathrm{CI}$ \\
\hline Intercept & 141.35 & 2.89 & & & $135.62,147.24$ \\
Condition (ME-E) & -0.03 & 1.19 & $\chi^{2}(1)=0.00$ & .98 & $-2.44,2.42$ \\
Time (30 min) & 17.32 & 1.14 & $\chi^{2}(1)=78.0$ & $<.001$ & $15.04,19.63$ \\
Sex (female-male) & 11.49 & 3.49 & $\chi^{2}(1)=8.9$ & .003 & $4.23,18.67$ \\
Condition*time & & & $\chi^{2}(1)=33.2$ & $<.001$ & \\
Intercept (ME-E) & 3.14 & 1.30 & & & $0.47,5.71$ \\
E*time & 20.08 & 1.22 & & & $17.63,22.42$ \\
(ME-E)*time & -5.43 & 0.92 & & & $-7.17,-3.63$ \\
\hline
\end{tabular}

Note. Intercepts in this model represents the estimate for E condition at time 0 for male participants. SE-standard error, $C I$ - confidence interval, $E$ - only exercise condition, $M E$ - combined mental rotation and exercise condition 
Table 3 Statistical Analysis of Heart Rate Without Exercise

\begin{tabular}{llllll}
\hline Variable & Estimate & SE & Test statistic & $\mathrm{p}$ & $95 \%$ CI \\
\hline Intercept & 84.36 & 2.04 & & & $80.01,88.28$ \\
Condition (baseline-M) & 0.13 & 1.89 & $\chi^{2}(1)=0.00$ & .95 & $-3.76,3.76$ \\
Time (30 min) & 2.48 & 1.34 & $\chi^{2}(1)=3.31$ & .07 & $-0.09,4.99$ \\
Sex (female-male) & 5.17 & 4.02 & $\chi^{2}(1)=1.60$ & .21 & $-2.31,13.50$ \\
\hline
\end{tabular}

Note. Intercepts in this model represents the estimate of the grand mean. Values for intercept and sex are from the model using both M condition and baseline. SE-standard error, CI-confidence interval, M-only mental rotation condition

showed significant differences (see Table 5 for inferential statistics and Fig. 4 for the descriptive comparison). The estimated difference of $\mathrm{pRPE}$ between conditions of -0.05 suggests no meaningful difference. For the comparison of sexes, the estimated difference of 0.15 might need further investigation.

\section{Mental Rotation}

Outliers were determined by a deviance of more than three standard deviations from the mean reaction time of all stimulus pairs with the same rotation angle and were excluded from all analyses. Because angular disparity is not defined for mirrored responses in cube figures (Shepard \& Metzler, 1971), only non-mirrored stimulus pairs were analyzed and reaction time was also only analyzed on correct responses.

\section{Reaction Time}

Model construction resulted in a model with random intercepts and slopes for condition, time and degree by participant, and random intercepts and slopes for time and degree by model. Time, condition, time*condition, angular disparity, and sex were analyzed as fixed effects and significant differences were found for angular disparity and time (see Table 6 for inferential statistics and Fig. 5 for the descriptive comparison of conditions and angular disparity). Reaction time improved significantly over time and significantly increased by degree. The estimates between both condition (27.45 ms faster in the $\mathrm{M}$ condition) and sexes (females $0.52 \mathrm{~ms}$ slower) are less than $1 \%$ of the average reaction time and suggest no meaningful differences.

\section{Accuracy}

Accuracy was analyzed by a general linear mixed model, which used a binomial distribution with random intercepts and slopes for time and degree by participant and random intercepts and slopes for time by model. Time, condition, time*condition, angular disparity, and sex were analyzed as fixed effects and significant differences were found for angular disparity and time (see Table 7 for inferential statistics and Fig. 6 for the descriptive comparison of conditions and angular disparity). Accuracy improved significantly over time and decreased significantly by degree. The point estimates for differences between condition and sex correspond to average changes in accuracy of $0.1 \%$ and $1.6 \%$ of trials, respectively. For the comparison of conditions in particular, this suggests no difference exists.

\section{Cognitive Subjective Effort}

For the analysis of the cRPE, the model building resulted in a model with only a random intercept by participant. Condition,
Table 4 Statistical Analysis of Pedal Cadence

\begin{tabular}{llllll}
\hline Variable & Estimate & $\mathrm{SE}$ & Test statistic & $\mathrm{p}$ & $95 \% \mathrm{CI}$ \\
\hline Intercept & 70.06 & 1.68 & & & $66.78,73.48$ \\
Condition (ME-E) & -8.32 & 1.85 & $\chi^{2}(1)=16.21$ & $<.001$ & $-11.82,-4.64$ \\
Time (30 min) & 9.20 & 1.59 & $\chi^{2}(1)=24.58$ & $<.001$ & $5.99,12.19$ \\
Sex (female-male) & -1.32 & 2.62 & $\chi^{2}(1)=0.23$ & .63 & $-6.69,3.84$ \\
Condition*time & & & $\chi^{2}(1)=77.59$ & $<.001$ & \\
Intercept (ME-E) & 0.25 & 2.06 & & & $-4.01,4.51$ \\
E*time & 16.60 & 1.81 & & & $12.70,20.22$ \\
(ME-E)*time & -14.76 & 1.58 & & & $-17.74,-11.49$ \\
\hline
\end{tabular}

Note. Intercepts in this model represents the estimate for E condition at time 0. SE- standard error, CIconfidence interval, $E$ - only exercise condition, $M E$ - combined mental rotation and exercise condition 


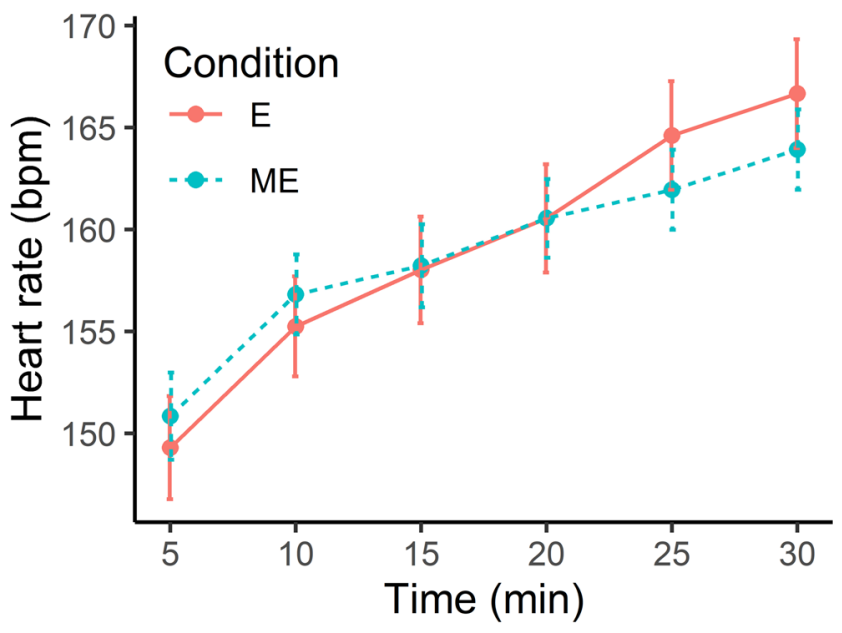

Note. Line plots showing mean pedal cadence as a function of time for both exercise conditions. Error bars show standard error. E - only exercise condition, $M E$ - combined mental rotation and exercise condition.

Fig. 3 Pedal Cadence During Exercise

sex, and their interaction were analyzed as fixed effects and we found significant differences for both condition and sex, but the interaction was not significant (see Table 8 for inferential statistics and Fig. 7 for the descriptive comparison). Subjective effort was significantly lower in women compared to men and in the M condition compared to $\mathrm{ME}$, both by approximately one value on the scale.

\section{Discussion}

The aim of the study was to explore the possible influences of simultaneous cognitive tasks on aerobic exercise and to further analyze the effects of exercise on complex cognitive function in the form of a mental rotation task. Our main results reveal no significant effects of neither simultaneous cognitive tasks on objective (heart rate) or subjective (RPE) physical effort nor of simultaneous exercise on reaction time or accuracy in cognitive performance. However, we identified lower cadence during the simultaneous mental rotation tasks; this was also stable in time compared to an increase in cadence

Table 5 Statistical Analysis of pRPE

\begin{tabular}{llllll}
\hline Variable & Estimate & SE & Test statistic & $\mathrm{p}$ & $95 \%$ CI \\
\hline Intercept & 6.35 & 0.15 & & & $6.06,6.64$ \\
Condition (ME-E) & -0.05 & 0.16 & $\chi^{2}(1)=0.10$ & .75 & $-0.36,0.24$ \\
Sex (female-male) & 0.15 & 0.30 & $\chi^{2}(1)=0.25$ & .62 & $-0.47,0.72$ \\
\hline
\end{tabular}

Note. Intercepts in this model represents the grand mean. SE-standard error, CI-confidence interval, E - only exercise condition, $M E$ - combined mental rotation and exercise condition

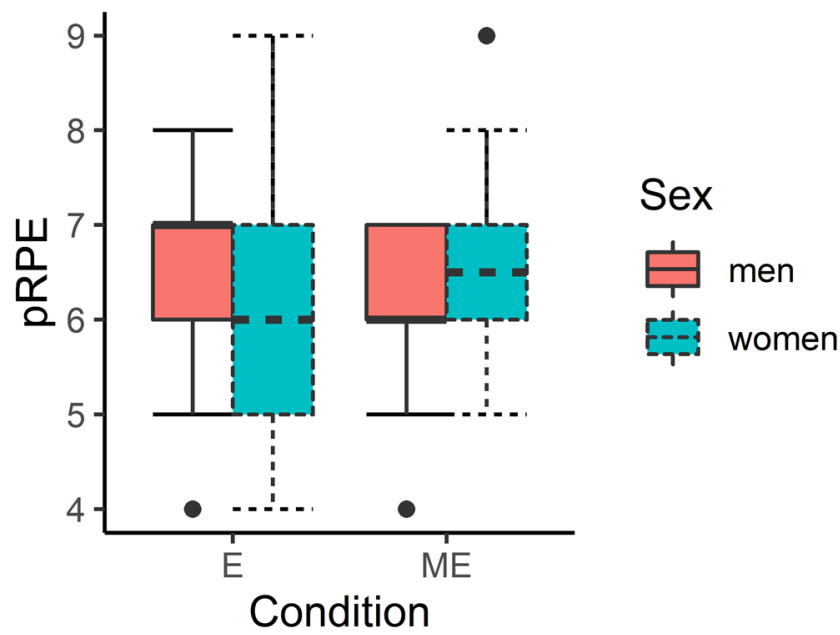

Note. Box plots showing pRPE after exercise conditions for both sexes. Whiskers are restricted in length to $1.5 * I Q R$. E - only exercise condition, $M E$ - combined mental rotation and exercise condition.

Fig. 4 PRPE After Exercise

during exercise control. Furthermore, our results demonstrated increased cognitive effort during exercise.

Since the increase in pedal cadence is linked to an increased physiological demand even at constant power, a lower heart rate should have been found during the cognitive tasks. Because the heart rate was comparable in the isolated exercise and the simultaneous exercise and mental rotation condition, this indicates that additional physiological effort was necessary in the combined condition due to the cognitive task. Similarly, the increased cognitive effort during exercise indicates that additional cognitive effort was necessary in the combined condition. Both can be interpreted in support of the RAH model (Dietrich \& Audiffren, 2011) and its hypothesized reverse direction, i.e. brain activity is not only caused by exercise, but contrary brain activity, as associated with cognitive function, also impacts exercise; as additional effort was necessary to maintain this performance, the tasks negatively impacted each other.

\section{Effects on Physical Performance}

The result of the lower cadence during the cognitive task could be explained by the assumption that the mental rotation task might stabilize cadence by serving as a distraction, while our control condition might subjectively be too boring and subsequently lead to increased cadence. While there is a linear relationship between power output and effort measured as heart rate or oxygen uptake (Arts \& Kuipers, 1994), there is evidence that this relationship is modulated by pedal cadence (Coast \& Welch, 1985; Faria et al., 2005a; MacIntosh et al., 2000). The optimal cadence, i.e. the cadence which produces the lowest effort at a given power output, increases linearly with power output (Coast \& Welch, 1985; MacIntosh et al., 2000) but might also be higher for trained cyclists (Faria et al., 2005a). Both higher and lower 
Table 6 Statistical Analysis of Reaction Time

\begin{tabular}{llllll}
\hline Variable & Estimate & SE & Test statistic & $\mathrm{p}$ & 95\% CI \\
\hline Intercept & 2310.59 & 127.06 & & & $2044.21,2559.32$ \\
Time (30 min) & -785.99 & 92.14 & $\chi^{2}(1)=37.61$ & $<.001$ & $-961.93,-601.83$ \\
Degree (100 $)$ & 1057.27 & 85.80 & $\chi^{2}(1)=55.91$ & $<.001$ & $895.61,1227.55$ \\
Sex (female-male) & 0.52 & 162.19 & $\chi^{2}(1)=0.00$ & .99 & $-315.69,338.95$ \\
Condition (M-ME) & -27.45 & 128.72 & $\chi^{2}(1)=0.05$ & .83 & $-266.64,248.64$ \\
Condition*time & & & $\chi^{2}(1)=0.65$ & .42 & \\
\hline
\end{tabular}

Note. Intercepts in this model represents the estimate at time 0 and unrotated stimuli. SE-standard error, CIconfidence interval, $M$ - only mental rotation condition, $M E$ - combined mental rotation and exercise condition than optimal cadences would lead to higher effort being exerted at given power outputs, i.e. a higher physiological demand despite the same energetical demand. However, without instruction or sufficient experience, people may be unable to ascertain their optimal pedal cadence and instead choose a higher than optimal cadence.

Research from Coast and Welch (1985) and MacIntosh et al. (2000) suggests that cadences of $70 \mathrm{rpm}$, as adopted on average in our ME condition, become optimal at around $250 \mathrm{~W}$. As $250 \mathrm{~W}$ is larger than any power implemented in our experimental conditions, this suggests higher than optimal cadence in both $\mathrm{E}$ and $\mathrm{ME}$ conditions. Because cadence was even higher in the E condition, one would expect even higher effort, both objectively and subjectively. As efforts were comparable between conditions, our results thus suggest that performing cognitive tasks while cycling has similar effects on heart rate and subjective effort as increasing cadence by a further 5-10 rpm above the optimal cadence, but the relationship between heart rate and subjective effort remains

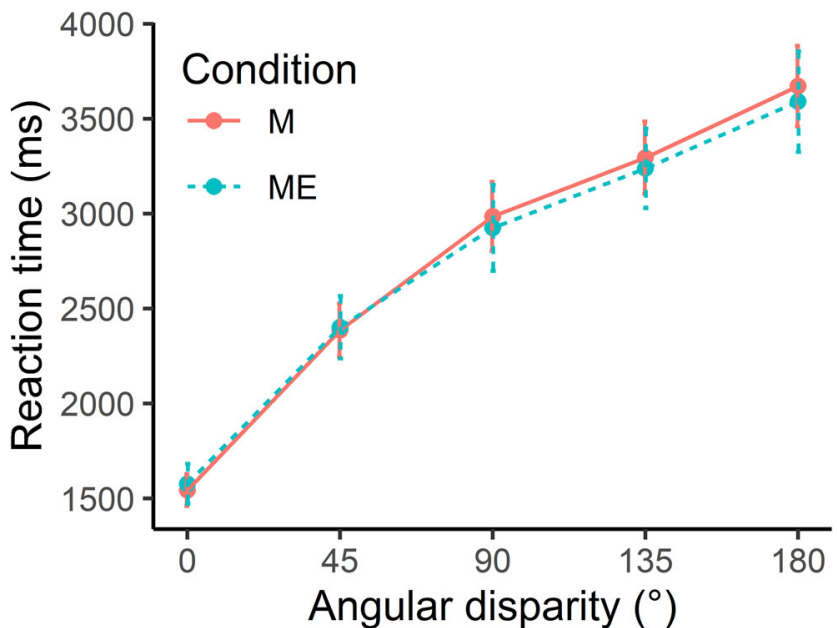

Note. Line plots showing mean reaction time of mental rotation trials as a function of angular disparity for both cognitive conditions. Mean reaction time is calculated for all non-mirrored and correctly answered trials of every participant and then averaged over all participants. Error bars show standard error. $M$ - only mental rotation condition, $M E$ - combined mental rotation and exercise condition.

Fig. 5 Reaction Time of Mental Rotation Tasks unchanged. Further research with better control of physiological demand is necessary to validate this relationship.

While the increase of heart rate over time can be explained by the differing cadence and the cardiovascular drift (the increase of heart rate over time during prolonged exercise, see e.g. Coyle \& González-Alonso, 2001), we have found a possible increase in heart rate caused by mental rotation alone in the resting condition. Thus, it is possible that mental rotation during aerobic exercise also causes a heart rate increase over time, just not by as much as in our exercise control condition due to the increasing cadence. However, due to the cardiovascular drift, the influence of the increase in cadence and the cognitive task on heart rate over time cannot be isolated.

Furthermore, we have compared resting cognitive heart rate to our baseline, which was measured under testing conditions in anticipation of exercise and found no difference. This suggests that even very light movement, like being strapped to a bicycle frame, the anticipation of exercise, or general nervousness from being tested, has a similar effect on heart rate as cognitive activity.

Our results suggest, in support of the reverse direction in the RAH model (Dietrich \& Audiffren, 2011), that a combination of cognitive with aerobic activity increases physical effort and thus might also affect physiological adaption, but an isolated effect of cognitive activity on physiology is

Table 7 Statistical Analysis of (Logarithmic Odds of) Accuracy

\begin{tabular}{llllll}
\hline Variable & Estimate & SE & Test statistic & $\mathrm{p}$ & $95 \% \mathrm{CI}$ \\
\hline Intercept & 3.56 & 0.23 & & & $3.14,4.01$ \\
Degree $\left(100^{\circ}\right)$ & -1.48 & 0.11 & $\chi^{2}(1)=68.15$ & $<.001$ & $-1.71,-1.27$ \\
Time $(30 \mathrm{~min})$ & 0.52 & 0.16 & $\chi^{2}(1)=9.89$ & .002 & $0.20,0.82$ \\
Condition & -0.03 & 0.04 & $\chi^{2}(1)=0.53$ & .47 & $-0.12,0.06$ \\
$\quad(M-M E)$ & & & & & \\
Sex (female-male) & -0.48 & 0.32 & $\chi^{2}(1)=2.22$ & .14 & $-1.04,0.10$ \\
Condition*time & & & $\chi^{2}(1)=0.02$ & .89 & \\
\hline
\end{tabular}

Note. Intercepts in this model represent the estimate for the logarithmic odds at time 0 and non-rotated stimuli. SE-standard error, CI-confidence interval, $M$ - only mental rotation condition, $M E$ - combined mental rotation and exercise condition 


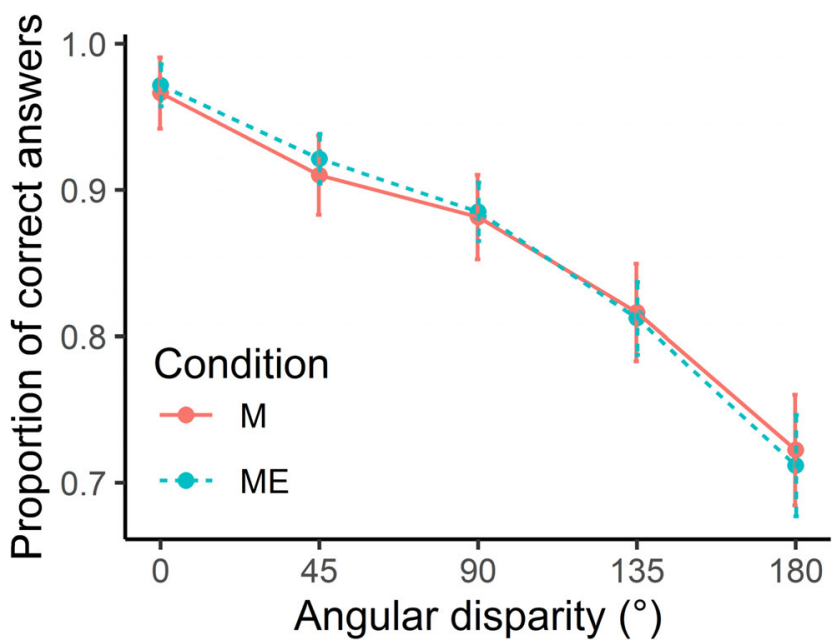

Note. Line plots showing mean accuracy of mental rotation trials as a function of angular disparity for both cognitive conditions. Mean accuracy is calculated for all non-mirrored trials of every participant and then averaged over all participants. Error bars show standard error. $M$ - only mental rotation condition, $M E$ - combined mental rotation and exercise condition.

Fig. 6 Accuracy of Mental Rotation Tasks

unlikely. As the relationship between subjective and objective effort does not change, it seems likely that cardiovascular adaptation remains steady if similar effort is invested. As workload might be lower in a combined activity of similar effort, skeletal muscular adaptations, while not being the primary target of aerobic training, might be influenced.

\section{Effects on Cognitive Performance}

We have found no effect of acute physical exercise on cognitive performance and no difference in improvement over the course of the units; however, we have discovered increased subjective cognitive effort. The measurement of cRPE might be influenced by the preceding measurement of pRPE in the $\mathrm{ME}$ condition, although we have found no correlation between physical and cognitive effort.

While reaction time and accuracy alone show no difference between conditions, nor any difference in temporal behavior, in contrast to the results of Lambourne and Tomporowski

Table 8 Statistical Analysis of cRPE

\begin{tabular}{llllll}
\hline Variable & Estimate & SE & Test statistic & $\mathrm{p}$ & $95 \% \mathrm{CI}$ \\
\hline Intercept & 6.29 & 0.30 & & & $5.71,6.87$ \\
Condition & -0.96 & 0.25 & $\chi^{2}(1)=12.26$ & $<.001$ & $-1.49,-0.45$ \\
$\quad($ M-ME) & & & & & \\
Sex (female-male) & -0.92 & 0.37 & $\chi^{2}(1)=5.88$ & .015 & $-1.63,-0.22$ \\
Condition*sex & & & $\chi^{2}(1)=0.12$ & .73 & \\
\hline
\end{tabular}

Note. Intercepts in this model represents the estimate in the base model for ME condition and male participants. SE- standard error, $C I-$ confidence interval, $M$ - only mental rotation condition, $M E$ - combined mental rotation and exercise condition

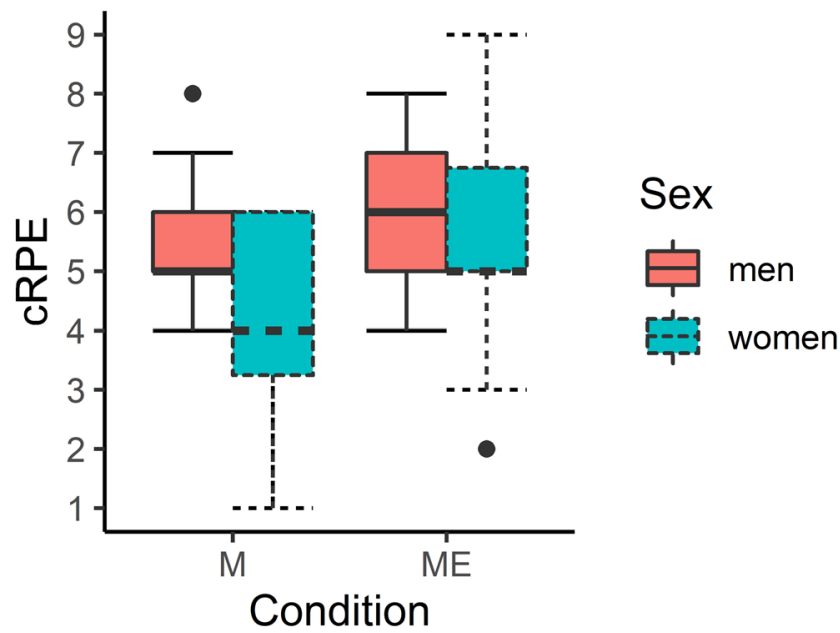

Note. Box plots showing cRPE after cognitive conditions for both sexes. Whiskers are restricted in length to $1.5 * I Q R$. $M$ - only mental rotation condition, $M E$ - combined mental rotation and exercise condition.

Fig. 7 CRPE After Mental Rotation

(2010) and Chang et al. (2012), the additional measurement of subjective effort allows for further interpretation. Subjective cognitive effort suggests that the mental rotation task was demanding but not maximal; after investing more effort, performance could be upheld under the more strenuous ME condition. While the assumption of a full depletion of self-control resources in the strength model (Audiffren \& André, 2015; Baumeister et al., 1998, 2007) might not have been fulfilled, results can be interpreted in support of the RAH model (Dietrich \& Audiffren, 2011). However, further objective measurements of effort and cognitive tasks that deplete neural resources should be employed in future experiments to support this claim.

\section{Sex Differences}

We have found increased heart rate in women during exercise and a lower cRPE in mental rotation, but no significant increase in pRPE. Increased heart rate can be explained by significant sex differences in performance test duration with lower durations and larger increases relative to maximal power leading to higher achieved maximal power. The missing concurrent increase in pRPE needs to be investigated further but could be explained by the choice of an overall lower RPE of the female participants, which can also be seen in the lower cRPE in cognitive tasks.

While the non-differences in mental rotation performance are in line with the results of Jansen-Osmann and Heil (2007), the lower cRPE is in contrast to the results of Campbell et al. (2018), who found increased cognitive effort in women using pupillometry. However, the different results could be explained by the varying measurement methods for the analysis of cognitive effort (subjective rating in this study in contrast to the measurement of cognitive strain in the study of Campbell 
et al., 2018). Further research is necessary to analyze the possible sex differences in selecting RPE and the potentially lower subjective effort for women in mental rotation tasks.

\section{Practical Implications}

The results indicate that increased effort, both physiologically and cognitively, is required during combined physical and cognitive work to maintain performance. Practical applications of a combination of aerobic exercise and cognitive tasks are for example learning during endurance training for athletes or treadmill and cycle desks to promote light physical activity during office work. While the performance could be maintained in our experiment, the increased effort could hinder adaptation of such a combination where long-term motivation is an issue. Moreover, further research is necessary if and to what extent the identified effects apply to highly fit individuals and low intensity exercise.

\section{Limitations}

It is possible that our results are restricted to specific parameters of both physical and cognitive performance and to the tested population of sport students. Limitations of the study also include possible changes in cognitive performance due to differences in upper body stability and in room conditions caused by physical exercise, as well as constant noise from the ergometer, which could be distracting but also drown out ambient noise. Although participants were asked to maintain their training, eating, and sleeping habits and mostly sustained their weekly and daily rhythms, these parameters were not explicitly monitored. Results could also be influenced by differences in emotional wellbeing (level of stress, anxiety, or depression), general fitness level, and time of testing between participants. The incentive to participate in this study was also through a seminar, which might affect results differently than the commonly used incentives of only study credit, monetary incentives, or wholly voluntary participation.

A technological limitation is the control of pedal cadence. As our participants did choose different cadences in the two conditions, the conclusions drawn rely on the relationship between cadence and physiological effort. While it is impossible to keep both power and pedal cadence constant at the same time and some control of power seems necessary, more comparable pedal cadences would be preferable to isolate the effects of cognitive tasks on physical effort.

Methodologically, it is possible that the transfer from the practice session to the rested condition was higher, as both were performed without aerobic exercise, although different seating conditions were used. Additionally, as RPE was only measured after the unit, time effects could only be observed in the objective parameters.

\section{Conclusion and Outlook}

In conclusion, we evidence that during simultaneous aerobic exercise and mental rotation in support of the RAH model (Dietrich \& Audiffren, 2011) subjective cognitive effort increases, while cognitive performance can be maintained. Moreover, in support of a possible reverse direction of the RAH model, physiological effort, mediated by pedal cadence, also increases. While the RAH model offers a rather simple explanation for the detected effects, further research is necessary to align the model with contradicting evidence. Measurements of effort might offer some insight into performance (non-)differences. Furthermore, for all future work employing simultaneous exercise and cognitive tasks we suggest employing a physical control condition in addition to the cognitive control condition.

However, to reinforce models like the RAH, brain image studies while participants perform a cognitive as well as a physical task have to be conducted. At this time, fNIRS seems to be the best neuroimaging method to obtain such measurements, but more advancements in this technology are necessary to accurately measure and interpret cerebral oxygenation and the hemodynamics of combined aerobic and cognitive tasks. Next to technological limitations and methodological concerns, possible complications also include physiological changes (e.g. heart rate), movement artifacts, and influences of posture (Herold et al., 2018).

Acknowledgements Data collection was supported by Arne Engelhardt (University of Regensburg).

Data and Code Availability The dataset generated and analyzed during the current study is available at https://osf.io/2m6wn/. Program code will be made available upon reasonable request.

Funding Open Access funding enabled and organized by Projekt DEAL. This research did not receive any specific grant from funding agencies in the public, commercial, or not-for-profit sectors.

\section{Declarations}

Ethics Approval The experiment was conducted according to ethical declaration of Helsinki. Ethical approval for this study was not required in accordance with the conditions outlined by the German Research Society (DFG) where research that carries no additional risk beyond daily activities does not require Research Ethics Board Approval. As the performance tests in this study were performed as part of a seminar there is no additional risk compared to the regular curriculum of sport students. We communicated all considerations necessary to assess the question of ethical legitimacy of the study.

Consent to Participate Informed consent was obtained from all individual participants included in the study.

Consent to Publish All participants consented to anonymous publication of their data. 
Conflict of Interest On behalf of all authors, the corresponding author states that there is no conflict of interest.

Open Access This article is licensed under a Creative Commons Attribution 4.0 International License, which permits use, sharing, adaptation, distribution and reproduction in any medium or format, as long as you give appropriate credit to the original author(s) and the source, provide a link to the Creative Commons licence, and indicate if changes were made. The images or other third party material in this article are included in the article's Creative Commons licence, unless indicated otherwise in a credit line to the material. If material is not included in the article's Creative Commons licence and your intended use is not permitted by statutory regulation or exceeds the permitted use, you will need to obtain permission directly from the copyright holder. To view a copy of this licence, visit http://creativecommons.org/licenses/by/4.0/.

\section{References}

Arts, F. J. P., \& Kuipers, H. (1994). The relation between power output, oxygen uptake and heart rate in male athletes. International Journal of Sports Medicine, 15(5), 228-231. https://doi.org/10.1055/s-20071021051

Audiffren, M., \& André, N. (2015). The strength model of self-control revisited: Linking acute and chronic effects of exercise on executive functions. Journal of Sport and Health Science, 4(1), 30-46. https:// doi.org/10.1016/j.jshs.2014.09.002.

Baguley, T. (2009). Standardized or simple effect size: What should be reported? British Journal of Psychology, 100(3), 603-617. https:// doi.org/10.1348/000712608X377117.

Barr, D. J., Levy, R., Scheepers, C., \& Tily, H. J. (2013). Random effects structure for confirmatory hypothesis testing: Keep it maximal. Journal of Memory and Language, 68(3), 255-278. https://doi. org/10.1016/j.jml.2012.11.001.

Bates, D., Kliegl, R., Vasishth, S., \& Baayen, H. (2015a). Parsimonious mixed models. ArXiv: 1506.04967. http://arxiv.org/abs/1506. 04967. Accessed 2020-10-30.

Bates, D., Mächler, M., Bolker, B. M., \& Walker, S. C. (2015b). Fitting linear mixed-effects models using lme4. Journal of Statistical Software, 67(1). https://doi.org/10.18637/jss.v067.i01.

Baumeister, R. F., Bratslavsky, E., Muraven, M., \& Tice, D. M. (1998). Ego depletion: Is the active self a limited resource? Journal of Personality and Social Psychology, 74(5), 1252-1265. https://doi. org/10.1037/0022-3514.74.5.1252.

Baumeister, R. F., Vohs, K. D., \& Tice, D. M. (2007). The strength model of self-control. Current Directions in Psychological Science, 16(6), 351-355. https://doi.org/10.1111/j.1467-8721.2007.00534.x.

Bentley, D. J., Newell, J., \& Bishop, D. (2007). Incremental exercise test design and analysis: Implications for performance diagnostics in endurance athletes. Sports Medicine, 37(7), 575-587 https://go. gale.com/ps/i.do?p=AONE \&sw=w\&issn=01121642\&v=2.1\&it= r\&id=GALE\%7CA200844739\&sid=googleScholar\&linkaccess= fulltext.

Bishop, P. A., \& Herron, R. L. (2015). Use and misuse of the Likert item responses and other ordinal measures. International Journal of Exercise Science, 8(3), 297-302 https://www.ncbi.nlm.nih.gov/ pmc/articles/PMC4833473/.

Brysbaert, M., \& Stevens, M. (2018). Power analysis and effect size in mixed effects models: A tutorial. Journal of Cognition, 1(1), 9. https://doi.org/10.5334/joc. 10 .

Büsch, D., Pabst, J., Naundorf, F., Braun, J., Marschall, F., Schumacher, K., Wilhelm, A., \& Granacher, U. (2015). Subjektive Beanspruchung im Krafttraining. In U. Granacher (Ed.), Krafttraining: "Kraftvoll durchs Leben": Jahrestagung der dvs-
Sektion Trainingswissenschaft vom 28.-30. Mai 2015 in Potsdam (Abstractband) (p. 13). Uni-Print.

Campbell, M. J., Toth, A. J., \& Brady, N. (2018). Illuminating sex differences in mental rotation using pupillometry. Biological Psychology, 138(February), 19-26. https://doi.org/10.1016/j. biopsycho.2018.08.003.

Chang, Y. K., Labban, J. D., Gapin, J. I., \& Etnier, J. L. (2012). The effects of acute exercise on cognitive performance: A meta-analysis. Brain Research, 1453(250), 87-101. https://doi.org/10.1016/j. brainres.2012.02.068.

Coast, J. R., \& Welch, H. G. (1985). Linear increase in optimal pedal rate with increased power output in cycle ergometry. European Journal of Applied Physiology and Occupational Physiology, 53(4), 339342. https://doi.org/10.1007/BF00422850.

Coyle, E. F., \& González-Alonso, J. (2001). Cardiovascular drift during prolonged exercise: New perspectives. Exercise and Sport Sciences Reviews, 29(2), 88-92. https://doi.org/10.1097/00003677200104000-00009.

Dietrich, A., \& Audiffren, M. (2011). The reticular-activating hypofrontality (RAH) model of acute exercise. Neuroscience and Biobehavioral Reviews, 35(6), 1305-1325. https://doi.org/10.1016/ j.neubiorev.2011.02.001.

Dodwe11, G., Müller, H. J., \& Töllner, T. (2019). Electroencephalographic evidence for improved visual working memory performance during standing and exercise. British Journal of Psychology, 110(2), 400-427. https://doi.org/10.1111/ bjop. 12352.

Faria, E. W., Parker, D. L., \& Faria, I. E. (2005a). The science of cycling: Factors affecting performance - part 2. In Sports Medicine (Vol. 35, issue 4, pp. 313-337). https://doi.org/10.2165/00007256200535040-00002

Faria, E. W., Parker, D. L., \& Faria, I. E. (2005b). The science of cycling: Physiology and training - Part 1. Sports Medicine, 35(4), 313-337. https://doi.org/10.2165/00007256-200535040-00003.

Faul, F., Erdfelder, E., Lang, A. G., \& Buchner, A. (2007). G*power 3: A flexible statistical power analysis program for the social, behavioral, and biomedical sciences. Behavior Research Methods, 39(2), 175191. https://doi.org/10.3758/BF03193146.

Feingold, A. (2009). Effect sizes for growth-modeling analysis for controlled clinical trials in the same metric as for classical analysis. Psychological Methods, 14(1), 43-53. https://doi.org/10.1037/ a0014699.Effect.

Hedges, L. V. (2007). Effect sizes in cluster-randomized designs. Journal of Educational and Behavioral Statistics, 32(4), 341-370. https:// doi.org/10.3102/1076998606298043.

Heil, M., \& Rolke, B. (2002). Toward a chronopsychophysiology of mental rotation. Psychophysiology, 39(4), 414-422. https://doi.org/ 10.1111/1469-8986.3940414

Herold, F., Wiegel, P., Scholkmann, F., \& Müller, N. (2018). Applications of functional near-infrared spectroscopy (fNIRS) neuroimaging in exercise-cognition science: A systematic, methodology-focused review. Journal of Clinical Medicine, 7(12), 466. https://doi.org/10.3390/jcm7120466.

Hilbert, S., Stadler, M., Lindl, A., Naumann, F., \& Bühner, M. (2019). Analyzing longitudinal intervention studies with linear mixed models. TPM - Testing, Psychometrics, Methodology in Applied Psychology, 26(1), 101-119. https://doi.org/10.4473/TPM26.1.6.

Hohmann, A., Lames, M., \& Letzelter, M. (2002). Einführung in die Trainingswissenschaft. Wiebelsheim: Limpert. https://opus. bibliothek.uni-augsburg.de/opus4/frontdoor/index/index/docId/ 34678. Accessed 2020-11-15.

Hopkins, W. G., Schabort, E. J., \& Hawley, J. A. (2001). Reliability of power in physical performance tests. In Sports Medicine (Vol. 31, issue 3, pp. 211-234). Adis international ltd. https://doi.org/10. 2165/00007256-200131030-00005. 
Hyun, J. S., \& Luck, S. J. (2007). Visual working memory as the substrate for mental rotation. Psychonomic Bulletin and Review, 14(1), 154 158. https://doi.org/10.3758/BF03194043.

Jansen, P., \& Pietsch, S. (2010). Physical activity improves mental rotation performance. Creative Education, 01(01), 58-61. https://doi. org/10.4236/ce.2010.11009.

Jansen, P., Titze, C., \& Heil, M. (2009). The influence of juggling on mental rotation performance. International Journal of Sport Psychology, 40(2), 351-359.

Jansen, P., Lehmann, J., \& van Doren, J. (2012). Mental rotation performance in male soccer players. PLoS One, 7(10), 1-7. https://doi.org/ 10.1371/journal.pone.0048620.

Jansen-Osmann, P., \& Heil, M. (2007). Suitable stimuli to obtain (no) gender differences in the speed of cognitive processes involved in mental rotation. Brain and Cognition, 64(3), 217-227. https://doi. org/10.1016/j.bandc.2007.03.002.

Jordan, K., Wüstenberg, T., Heinze, H. J., Peters, M., \& Jäncke, L. (2002). Women and men exhibit different cortical activation patterns during mental rotation tasks. Neuropsychologia, 40(13), 23972408. https://doi.org/10.1016/S0028-3932(02)00076-3.

Kahneman, D., Tursky, B., Shapiro, D., \& Crider, A. (1969). Pupillary, heart rate, and skin resistance changes during a mental task. Journal of Experimental Psychology, 79(1, Pt.1), 164-167. https://doi.org/ $10.1037 / \mathrm{h} 0026952$

Kennedy, D. O., \& Scholey, A. B. (2000). Glucose administration, heart rate and cognitive performance: Effects of increasing mental effort. Psychopharmacology, 149(1), 63-71. https://doi.org/10.1007/ s002139900335.

Lambourne, K., \& Tomporowski, P. (2010). The effect of exerciseinduced arousal on cognitive task performance: A meta-regression analysis. Brain Research, 1341, 12-24. https://doi.org/10.1016/j. brainres.2010.03.091.

MacIntosh, B. R., Neptune, R. R., \& Horton, J. F. (2000). Cadence, power, and muscle activation in cycle ergometry. Medicine and Science in Sports and Exercise, 32(7), 1281-1287. https://doi.org/ 10.1097/00005768-200007000-00015.

Martin, K., Meeusen, R., Thompson, K. G., Keegan, R., \& Rattray, B. (2018). Mental fatigue impairs endurance performance: A physiological explanation. Sports Medicine, 48(9), 2041-2051. https://doi. org/10.1007/s40279-018-0946-9.

Moè, A., Jansen, P., \& Pietsch, S. (2018). Childhood preference for spatial toys. Gender differences and relationships with mental rotation in STEM and non-STEM students. Learning and Individual Differences, 68, 108-115. https://doi.org/10.1016/j.lindif.2018.10. 003.

Oberste, M., Javelle, F., Sharma, S., Joisten, N., Walzik, D., Bloch, W., \& Zimmer, P. (2019). Effects and moderators of acute aerobic exercise on subsequent interference control: A systematic review and Metaanalysis. In Frontiers in Psychology (Vol. 10). Frontiers media S.A. https://doi.org/10.3389/fpsyg.2019.02616.

Oldfield, R. C. (1971). The assessment and analysis of handedness: The Edinburgh inventory. Neuropsychologia, 9(1), 97-113. https://doi. org/10.1016/0028-3932(71)90067-4.
Pek, J., \& Flora, D. B. (2018). Reporting effect sizes in original psychological research: A discussion and tutorial. Psychological Methods, 23(2), 208-225. https://doi.org/10.1037/met0000126.

Peters, M., \& Battista, C. (2008). Applications of mental rotation figures of the Shepard and Metzler type and description of a mental rotation stimulus library. Brain and Cognition, 66(3), 260-264. https://doi. org/10.1016/j.bandc.2007.09.003.

Pietsch, S., Böttcher, C., \& Jansen, P. (2017). Cognitive motor coordination training improves mental rotation performance in primary school-aged children. Mind, Brain, and Education, 11(4), 176180. https://doi.org/10.1111/mbe.12154.

R Core Team. (2018). R: A Language and Environment for Statistical Computing. https://www.r-project.org/. Accessed 2020-10-05.

Rights, J. D., \& Sterba, S. K. (2019). Quantifying explained variance in multilevel models: An integrative framework for defining R-squared measures. Psychological Methods, 24(3), 309-338. https://doi.org/ 10.1037/met0000184.

Schaefer, S., \& Schumacher, V. (2011). The interplay between cognitive and motor functioning in healthy older adults: Findings from dualtask studies and suggestions for intervention. Gerontology, 57(3), 239-246. https://doi.org/10.1159/000322197.

Schaefer, S., Jagenow, D., Verrel, J., \& Lindenberger, U. (2015). The influence of cognitive load and walking speed on gait regularity in children and young adults. Gait and Posture, 41(1), 258-262. https://doi.org/10.1016/j.gaitpost.2014.10.013.

Shepard, R. N., \& Metzler, J. (1971). Mental rotation of threedimensional objects. Science, 171(3972), 701-703. https://doi.org/ 10.1126/science.171.3972.701.

Such, U., \& Meyer, T. (2010). Die maximale herzfrequenz. Deutsche Zeitschrift fur Sportmedizin, 61(12), 310-311.

Van Cutsem, J., Marcora, S., De Pauw, K., Bailey, S., Meeusen, R., \& Roelands, B. (2017). The effects of mental fatigue on physical performance: A systematic review. Sports Medicine, 47(8), 1569-1588. https://doi.org/10.1007/s40279-016-0672-0.

Voyer, D., \& Jansen, P. (2017). Motor expertise and performance in spatial tasks: A meta-analysis. Human Movement Science, 54, $110-124$.

Voyer, D., Voyer, S., \& Bryden, M. P. (1995). Magnitude of sex differences in spatial abilities: A meta-analysis and consideration of critical variables. Psychological Bulletin, 117(2), 250-270. https://doi. org/10.1037/0033-2909.117.2.250.

Wohlschläger, A., \& Wohlschläger, A. (1998). Mental and manual rotation. Journal of Experimental Psychology: Human Perception and Performance, 24(2), 397-412. https://doi.org/10.1037/0096-1523. 24.2.397.

Xie, F., Zhang, L., Chen, X., \& Xin, Z. (2020). Is spatial ability related to mathematical ability: A Meta-analysis. Educational Psychology Review, 32(1), 113-155. https://doi.org/10.1007/s10648-01909496-y.

Publisher's Note Springer Nature remains neutral with regard to jurisdictional claims in published maps and institutional affiliations. 